Kurz notiert

Dialyse und Herzinsuffizienz

Weniger Todesfälle durch $\mathrm{AT}_{1}$-Rezeptorblocker

Bei Dialysepatienten mit Herzinsuffizienz konnte die Behandlung mit dem $\mathrm{AT}_{1}$-Rezeptorblocker Telmisar$\tan \left(\right.$ Micardis ${ }^{\circledR}$ ) zusätzlich zur Standardtherapie mit einem ACE-Hemmer die Lebenserwartung dieser Patienten dramatisch verbessern. In einer italienischen Studie mit 332 Patienten wurde die Gesamtsterberate innnerhalb von drei Jahren durch Telmisartan signifikant um 49\% im Vergleich zu Placebo reduziert (von 54 auf 35\%). Entscheidend dafür war die Verhinderung von kardiovaskulären Todesfällen, deren Rate signifikant um $58 \%$ gesenkt wurde (von 44 auf 30\%).

II Boehringer Ingelheim

Mit Platin-Chrom-Legierung Stent der dritten Generation

Im Elisabeth-Krankenhaus in Essen wurde kürzlich erstmals das unbeschichtete Koronarstentsystem OMEGA $^{\mathrm{TM}}$ implantiert. Das Besondere an diesem Stent ist seine PlatinChrom-Legierung, die für eine bessere Performance im Vergleich zu herkömmlichen Stents sorgt.

II Boston Scientific

Positiv beurteilt

Direkter Reninhemmer plus Kalziumantagonist

Den Antrag auf Zulassung einer Fixkombination aus dem direkten Reninhemmer Aliskiren und dem Kalziumkanalblocker Amlodipin hat das europäische Committee for Medicinal Products for Human Use (CHMP) nun positiv bewertet. Von dieser Kombination profitieren vor allem Hypertoniker mit Diabetes und Übergewicht.

II Novartis

\title{
Typ-2-Diabetes
}

\section{Sitagliptin so effektiv wie Pioglitazon}

Bei unbehandeltem Typ-2-Diabetes haben Sitagliptin und Pioglitazon im direkten Vergleich die Blutzuckerkontrolle gleichermaßen klinisch signifikant verbessert. Der DPP-4-Hemmer bietet sich deshalb als Alternative an, wenn ab April das Glitazon nicht mehr uneingeschränkt zu Lasten der GKV verordnet werden kann.

An der randomisierten Studie nahmen 492 Typ-2-Diabetiker mit moderater bis schwerer Hyperglykämie teil. Nach der ersten Doppelblindphase über zwölf Wochen unter der Monotherapie mit Sitagliptin 100 mg/ Tag oder Pioglitazon $15 \mathrm{mg} / \mathrm{Tag}$ (nach sechs Wochen hochtitriert auf $30 \mathrm{mg}$ ) war der $\mathrm{HbA}_{1 \mathrm{C}}$-Wert in beiden Gruppen vergleichbar gesunken (um -1\% bzw. $-0,9 \%$ ).

\section{Vergleichbare Reduktion des $\mathrm{HbA}_{1 \mathrm{c}}$} In einer zweiten Studienphase von 28 Wochen wurde Sitagliptin durch die Fixkombination Sitagliptin/Metformin ersetzt, in der Vergleichsgruppe wurde Pioglitazon auf 45 $\mathrm{mg} /$ Tag hochtitriert. Am Ende dieser Phase war der Therapieerfolg unter Sitagliptin/ Metformin signifikant stärker ausgeprägt. Der $\mathrm{HbA}_{1 \mathrm{c}}$-Wert war um $1,8 \%$ (vs. 1,4\%) gesunken, und $55 \%$ der Patienten (vs. 40,5\% unter Pioglitazon) lagen unter dem $\mathrm{HbA}_{1 \mathrm{C}^{-}}$ Zielwert von $7 \%$.

\section{Gute Verträglichkeit}

Die Verträglichkeit war in beiden Behandlungsgruppen durchweg gut. Hypoglykämie-Episoden spielten keine wesentliche Rolle (Inzidenz: 2,3\% bzw. 2,2\%). Unter Sitagliptin/Metformin traten signifikant weniger Ödeme auf (0,9 vs. 6.1\%), unter Pioglitazon seltener gastrointestinale Nebenwirkungen.

Als wesentlicher Vorteil der SitagliptinMetformin-Therapie erwies sich die Gewichtsabnahme von im Mittel 1,1 kg, während die Patienten unter Pioglitazon im Mittel 3,4 kg zulegten.

EE I

II Pérez-Monteverde A et al. Diabetologia 2010:53 (Suppl. 1) S325

\section{Koronare Herzkrankheit}

\section{Akut-Nitrat zur Unterstützung der Rehabilitation}

\section{Akut-Nitrate sind Standard bei der Behandlung von Angina-pectoris- Anfällen. Darüber hinaus können sie auch zu einer effektiveren Rehabilitation von Patienten mit koronarer Herzkrankheit (KHK) beitragen.}

Gemäß der nationalen Versorgungsleitlinie "Chronische KHK" sollten Patienten mit stabiler Angina pectoris stets ein schnell wirkendes Nitrat zur Kupierung akuter Anfälle und zur Anfallsprophylaxe zur Hand haben. Denn auch unter optimaler medikamentöser Therapie wird die Häufigkeit der AnginaSymptomatik unterschätzt .

\section{Beschwerden trotz optimaler medikamentöser Therapie}

Die CADENCE (Coronary Artery Disease in General Practice)-Studie zeigte, dass 48\% der KHK-Patienten, die nach ärztlicher Einschätzung optimal medikamentös eingestellt waren, dennoch pektanginöse Beschwerden hatten, $7 \%$ davon sogar täglich (Beltrame JF et al. Arch Intern Med. 2009;169: 1491-1499).

Prophylaxe durch körperliches Training Die European Association of Cardiovascular Prevention and Rehabilitation (EACPR) empfiehlt bei Patienten mit stabiler KHK, die eine medikamentöse Therapie erhalten und/oder 